University of Wollongong

Research Online

Faculty of Engineering and Information

Faculty of Engineering and Information

Sciences - Papers: Part B

Sciences

2019

Variance and volatility swaps under a two-factor stochastic volatility model with regime switching

Xinjiang He

University of Wollongong, xinjiang@uow.edu.au

Song-Ping Zhu

University of Wollongong, spz@uow.edu.au

Follow this and additional works at: https://ro.uow.edu.au/eispapers1

Part of the Engineering Commons, and the Science and Technology Studies Commons

Research Online is the open access institutional repository for the University of Wollongong. For further information contact the UOW Library: research-pubs@uow.edu.au 


\title{
Variance and volatility swaps under a two-factor stochastic volatility model with regime switching
}

\author{
Abstract \\ In this paper, the pricing problem of variance and volatility swaps is discussed under a two-factor \\ stochastic volatility model. This model can be treated as a two-factor Heston model with one factor \\ following the CIR process and another characterized by a Markov chain, with the motivation originating \\ from the popularity of the Heston model and the strong evidence of the existence of regime switching in \\ real markets. Based on the derived forward characteristic function of the underlying price, analytical \\ pricing formulae for variance and volatility swaps are presented, and numerical experiments are also \\ conducted to compare swap prices calculated through our formulae and those obtained under the Heston \\ model to show whether the introduction of the regime switching factor would lead to any significant \\ difference. \\ Disciplines \\ Engineering | Science and Technology Studies

\section{Publication Details} \\ He, X. \& Zhu, S. (2019). Variance and volatility swaps under a two-factor stochastic volatility model with \\ regime switching. International Journal of Theoretical and Applied Finance, 22 (4), \\ 1950009-1-1950009-19.
}




\title{
Variance and volatility swaps under a two-factor stochastic volatility model with regime switching
}

\author{
Xin-Jiang $\mathrm{He}^{*} \quad$ Song-Ping Zhu ${ }^{\dagger}$
}

\begin{abstract}
In this paper, the pricing problem of variance and volatility swaps is discussed under a two-factor stochastic volatility model. This model can be treated as a two-factor Heston model with one factor following the CIR process and another characterized by a Markov chain, with the motivation originating from the popularity of the Heston model and the strong evidence of the existence of regime switching in real markets. Based on the derived forward characteristic function of the underlying price, analytical pricing formulae for variance and volatility swaps are presented, and numerical experiments are also conducted to compare swap prices calculated through our formulae and those obtained under the Heston model to show whether the introduction of the regime switching factor would lead to any significant difference.
\end{abstract}

\section{AMS(MOS) subject classification.}

Keywords. Variance swaps, volatility swaps, stochastic volatility, regime switching, Heston model, CIR model, Markov chains.

\footnotetext{
* School of Mathematics and Applied Statistics, University of Wollongong NSW 2522, Australia.

${ }^{\dagger}$ Corresponding author. School of Mathematics and Applied Statistics, University of Wollongong NSW 2522, Australia. Email: spz@uow.edu.au. Telephone: +61242213807.
} 


\section{Introduction}

Volatility derivatives are becoming increasingly popular due to the fact that volatility is often used as a measure of risk, and there are large demands for managing financial risk caused by the high trading volumes of different kinds of financial derivatives. In particular, variance and volatility swaps, whose pay-off is dependent on the future realized variance and volatility respectively, are two of the most popular volatility derivative contracts, and thus considerable research effort is devoted to the area of accurately pricing the two contracts.

In fact, there are mainly two approaches in determining the price of variance and volatility swaps, depending on the sampling method of the realized variance or volatility. Specifically, the first is based on the assumption of continuous sampling, which has already attracted a number of researchers. Within this category, various stochastic volatility models have been adopted (Grünbichler \& Longstaff 1996, Howison et al. 2004, Javaheri et al. 2004, Swishchuk 2004, Salvi \& Swishchuk 2014). Swishchuk (2006) went even further to consider the effect of the delayed response observed in real markets in the pricing of variance and volatility swaps. Moreover, jumps, as another common feature shown by different underlying asset prices, have already been incorporated in the pricing dynamics (Habtemicael \& Sengupta 2016a, Habtemicael \& SenGupta 2016b, Issaka \& SenGupta 2017), while some general model independent results were also presented by Carr \& Lee (2007, 2008). Unfortunately, the realized variance or volatility is actually discretely sampled in real markets, which implies that there would always exist biases or even large errors if we use the continuous sampled results as approximation, and thus it is much more important to take into consideration the pricing problem of discretely-sampled variance and volatility swaps.

Recently, a few studies on discretely sampled variance and volatility swaps have been conducted so that the pricing results can be directly applied in reality. By adopting the dimension reduction techniques, Zhu \& Lian (2011) derived a closed-form pricing formula for 
variance swaps under the Heston stochastic volatility model for the first time. In addition, the analytical solution for volatility swap prices under the Heston model (Heston 1993) has also been found in Zhu \& Lian (2015). One may argue that there is no need to work on this area since the Heston model is very popular in real markets and analytical formulae for the two contracts have already been derived under this particular model. However, it needs to be noted that the Heston model is not perfect either, and there also exists some model flaws (Byelkina \& Levin 2010), which implies that the Heston model alone is not enough and other models may be more suitable for certain markets. More importantly, the Heston model does not incorporate the mechanics of regime switching, which sometimes is at odds with empirical evidence strongly demonstrating the existence of regime switching in the underlying price (Eraker 2004, Hamilton 1990). This prompts the research in developing Markov-modulated models. A typical example is that variance and volatility swaps are analytically priced under the Heston model with the mean-reversion level of the stochastic volatility being assumed to be regime switching (Elliott \& Lian 2013). Moreover, He \& Zhu (2016) went even further by empirically comparing the performance of the Heston model and their newly proposed regime switching Heston model in option pricing, and results demonstrated that the incorporation of regime switching into the stochastic volatility model can indeed provide better fit to market data in certain cases. Recently, local regime-switching models have also been introduced (Elliott et al. 2015) and some relevant issues were discussed in He \& Zhu (2017, 2018).

In this paper, we adopt a two-factor stochastic volatility model, with one factor modeled by the CIR process, the same as that in the Heston model, and another being controlled by a Markov chain. It should be pointed out here that in the following we shall focus on discussing the two-state regime-switching model for illustration purpose, and the extension to arbitrary but finite number of states should be in principle very similar to the results presented here. In the literature, two-factor stochastic volatility models with regime switching have already been considered by some authors. For instance,the pricing problem 
of European options under the same model in considered in Kun (2014), while Siu et al. (2008) adopted a two-factor model with one following a log-normal diffusion process and another characterized by a Markov chain to price currency options. In the following, we first derive a closed-form formula for the forward characteristic function of the underlying price, based on which the analytical formulae for variance and volatility swap prices are presented. Numerical experiments are subsequently carried out to study the influence of introducing the new regime switching factor into the Heston stochastic volatility model.

The rest of the paper is organized as follows. In Section 2, we will firstly introduce the two-factor stochastic volatility model, and then analytically solutions for variance and volatility swap prices are presented with the inverse Fourier transform. In Section 3, numerical examples are presented, followed by some concluding remarks given in the last section.

\section{Closed-form solution}

In this section, a two-factor stochastic volatility model will be firstly introduced with one factor following the CIR process, which is the same as the Heston model, and another being controlled by a Markov chain. The motivation for using such a model is a lot of empirical evidence demonstrating that the dynamics of the underlying price are better captured with the regime switching mechanics (Eraker 2004, Hamilton 1990). Then, an

analytical solution for the forward characteristic function of the underlying price will be presented, based on which the closed-form pricing formulae for variance and volatility swaps are straightforwardly derived.

\subsection{The two-factor stochastic volatility model}

Given the fact that the adopted two-factor stochastic volatility model can be viewed as a two-factor Heston model, and the Heston model is a well-known stochastic volatility model 
and widely used even in today's financial markets, we would like introduce the well-known model in the first place, the dynamics of which are specified as

$$
\begin{aligned}
\frac{d S_{t}}{S_{t}} & =r d t+\sqrt{v_{t}} d W_{t}^{1} \\
d v_{t} & =k\left(\theta-v_{t}\right) d t+\sigma \sqrt{v_{t}} d W_{t}^{2}
\end{aligned}
$$

where $\left\{S_{t}\right\}_{t \geq 0}$ and $\left\{v_{t}\right\}_{t \geq 0}$ represent the underlying price and the volatility respectively. $\left\{W_{t}^{1}\right\}_{t \geq 0}$ and $\left\{W_{t}^{2}\right\}_{t \geq 0}$ are the two standard Brownian motions with correlation $\rho$. $k$ and $\theta$ denote the mean reverting speed and the long-term mean respectively, while $\sigma$ is the volatility of volatility.

Having presented the Heston model, we are now ready to introduce the one we adopt, which is

$$
\begin{aligned}
\frac{d S_{t}}{S_{t}} & =r d t+\sqrt{v_{t}} d W_{t}^{1}+\alpha_{X_{t}} d B_{t} \\
d v_{t} & =k\left(\theta-v_{t}\right) d t+\sigma \sqrt{v_{t}} d W_{t}^{2}
\end{aligned}
$$

with $\left\{B_{t}\right\}_{t \geq 0}$ being another standard Brownian motion independent of the other two standard Brownian motions $\left\{W_{t}^{1}\right\}_{t \geq 0}$ and $\left\{W_{t}^{2}\right\}_{t \geq 0}$. The two-state Markov chain $\left\{X_{t}\right\}_{t \geq 0}$ is independent of the three Brownian motions defined as

$$
X_{t}= \begin{cases}(1,0)^{\prime}, & \text { when the economy is believed to be in state } 1 \\ (0,1)^{\prime}, & \text { when the economy is believed to be in state } 2\end{cases}
$$

with the dash denoting transposition. The transition between the two states follows a Poisson process as

$$
P\left(t_{i j}>t\right)=e^{-\lambda_{i j} t}, i, j=1,2, i \neq j
$$

where $\lambda_{i j}$ is the transition rate from state $i$ to $j$, and $t_{i j}$ is the time spent in state $i$ before transferring to state $j$. $\alpha_{X_{t}}$ can be determined through $\alpha_{X_{t}}=\left\langle\bar{\alpha}, X_{t}\right\rangle$ with $\bar{\alpha}=\left(\alpha_{1}, \alpha_{2}\right)^{\prime}$ 
and $\langle\cdot, \cdot\rangle$ being the inner product of two vectors.

It should be pointed out here that when $\alpha_{1}=\alpha_{2}=0$, our two-factor model will degenerate to the Heston model, which implies that our model is more general than the Heston model with an enlarged parameter space. This allows the stochastic nature of the underlying volatility being not only described by the CIR process, but also controlled by the regime switching mechanics, which are also a common feature observed in real markets (Eraker 2004, Hamilton 1990).

\subsection{Valuation of variance and volatility swaps}

To determine the price of variance and volatility swaps, we should firstly figure out how "price" is defined. As known to us all that variance and volatility swaps are two kinds of forward contracts, the price to be derived is actually the delivery price specified in the contracts, instead of being the value of the contracts. In fact, the pay-off function of variance and volatility swaps also depends on the future realized variance and volatility respectively, and the long position of variance or volatility swaps pays the delivery price at expiry in exchange of the floating amount of the realized variance or volatility within the time period of the contract.

If we take a careful look into the variance and volatility swaps, it is not difficult to find that the values of the two contracts can be respectively expressed as

$$
V_{v a r}=\left(R V_{v a r}-K_{v a r}\right) L, \quad V_{v o l}=\left(R V_{v o l}-K_{v o l}\right) L
$$

with $L$ being the notional amount. $R V_{v a r}$ and $R V_{v o l}$ denote the annualized realized variance and volatility respectively, and $K_{v a r}$ and $K_{v o l}$ are the corresponding delivery price of a variance and volatility swap contract that need to be determined, respectively. If we take into consideration the fact that the value of a forward contract should equal to zero when 
it is initialized in order to be fair to both parties, we can certainly obtain

$$
K_{v a r}=E\left[R V_{v a r}\right], \quad K_{v o l}=E\left[R V_{v o l}\right]
$$

which shows that the definition of the realized variance and volatility really matters when we try to price variance and volatility swaps. In the existing literature, one of the most widely adopted definitions for the realized variance and volatility (Elliott \& Lian 2013, Howison et al. 2004, Zhu \& Lian 2011) are respectively

$$
\begin{aligned}
R V_{v a r} & =\frac{c^{2}}{T} \sum_{i=1}^{N}\left(\frac{S_{t_{i}}-S_{t_{i-1}}}{S_{t_{i-1}}}\right)^{2}, \\
R V_{\text {vol }} & =c \sqrt{\frac{\pi}{2 N T}} \sum_{i=1}^{N}\left|\frac{S_{t_{i}}-S_{t_{i-1}}}{S_{t_{i-1}}}\right|,
\end{aligned}
$$

where $T$ is the expiry time, and the time period $[0, T]$ is uniformly discretized into $N$ small periods $\left[t_{i-1}, t_{i}\right]$ for $i=1,2, . ., N$. The constant $c=100$ is the conversion factor between the actual strike price and the market quoted strike price (with \% sign) of a variance/volatility swap $^{1}$. Therefore, the calculation of swap prices is reduced to the evaluation of the $2 N$ expectations, i.e. $E\left[\left(\frac{S_{t_{i}}-S_{t_{i-1}}}{S_{t_{i-1}}}\right)^{2}\right]$ and $E\left[\left|\frac{S_{t_{i}}-S_{t_{i-1}}}{S_{t_{i-1}}}\right|\right]$, the expression of which clearly shows that once the forward characteristic function of the underlying price is analytically worked out, these expectations as well as the target swap prices can be straightforwardly derived. To be more specific, the forward characteristic function is defined as

$$
m\left(\phi ; t, T, y_{0}, v_{0}, X_{0}\right)=E\left[e^{j \phi y_{T}} \mid y_{0}, v_{0}, X_{0}\right]
$$

where $y_{T}=\ln \left(S_{T}\right)-\ln \left(S_{t}\right)$. Due to the existence of the Markov chain, it is very difficult to directly calculate the value of $m\left(\phi ; t, T, y_{0}, v_{0}, X_{0}\right)$, and thus we alternatively try to find the solution of the conditional forward characteristic function first, which is conditional

\footnotetext{
${ }^{1}$ It should be noted in passing that this conversion factor is mistakenly not shown in Equation (9) of Zhu \& Lian (2012), just as it has appeared in all the rest of the equations prior to Equation (9).
} 
upon all the information of the Markov chain during the lifetime of the swap contract being known at the current time, i.e.,

$$
m\left(\phi ; t, T, y_{0}, v_{0} \mid X_{T}\right)=E\left[e^{j \phi y_{T}} \mid y_{0}, v_{0}, X_{T}\right]
$$

The solution of this conditional characteristic function can be obtained since $\alpha_{X_{t}}$ only needs to be treated as a time-dependent function $\alpha_{t}$, the results of which are presented in the following proposition.

Proposition 2.1. Let the underlying price $S_{t}$ and the volatility $v_{t}$ follow the dynamics specified in Equation (2.2), the conditional forward characteristic function $m\left(\phi ; t, T, v_{0} \mid X_{T}\right)$ can be derived as

$$
m\left(\phi ; t, T, v_{0} \mid X_{T}\right)=e^{\widetilde{C}(\phi ; \tau, t)+\bar{D}(\phi ; \tau, t) v_{0}} e^{\int_{t}^{T}\left\langle-\frac{1}{2}\left(j \phi+\phi^{2}\right) \bar{\alpha}^{2}, X_{s}\right\rangle d s}
$$

where

$$
\begin{aligned}
\bar{D}(\phi ; \tau, t) & =\frac{2 k}{\sigma^{2}} \frac{1}{1-\left[1-\frac{2 k}{\sigma^{2} D(\phi ; \tau)}\right] e^{k t}}, \\
\widetilde{C}(\phi ; \tau, t) & =\bar{C}(\phi ; \tau, t)+r j \phi \tau+\frac{k \theta}{\sigma^{2}}\left\{[d-(j \phi \rho \sigma-k)] \tau-2 \ln \left[\frac{1-g e^{d \tau}}{1-g}\right]\right\}, \\
\bar{C}\left(\phi ; \tau, \tau_{s}\right) & =\frac{2 k \theta}{\sigma^{2}}\left\{k \tau_{s}-\ln \left[1-\left(1-\frac{2 k}{\sigma^{2} D}\right) e^{k \tau_{s}}\right]+\ln \left(\frac{2 k}{\sigma^{2} D}\right)\right\}, \\
D(\phi ; \tau) & =\frac{d-(\rho \sigma j \phi-k)}{\sigma^{2}} \frac{1-e^{d \tau}}{1-g e^{d \tau}}, \\
d & =\sqrt{(\rho \sigma j \phi-k)^{2}+\sigma^{2}\left(j \phi+\phi^{2}\right)}, \quad g=\frac{(\rho \sigma j \phi-k)-d}{(\rho \sigma j \phi-k)+d},
\end{aligned}
$$

with $\tau=T-t, j=\sqrt{-1}, X_{t} \in\left\{(1,0)^{\prime},(0,1)^{\prime}\right\}$, and $\langle\cdot, \cdot\rangle$ denotes the inner product of two vectors. Note: it turns out that $y_{0}$ is not a parameter in the expression of $m\left(\phi ; t, T, v_{0} \mid X_{T}\right)$, and it is thus omitted.

Proof. According to the tower rule of the expectation, the conditional forward character- 
istic function can be calculated as

$$
\begin{aligned}
m\left(\phi ; t, T, y_{0}, v_{0} \mid X_{T}\right) & =E\left[e^{j \phi y_{T}} \mid y_{0}, v_{0}, X_{T}\right] \\
& =E\left[E\left(e^{j \phi y_{T}} \mid y_{t}, v_{t}, X_{T}\right) \mid y_{0}, v_{0}, X_{T}\right]
\end{aligned}
$$

which implies that the inner expectation must be worked out before we can obtain the final solution. If we assume

$$
h\left(\phi ; s, T, y_{s}, v_{s} \mid X_{T}\right)=E\left(e^{j \phi y_{T}} \mid y_{s}, v_{s}, X_{T}\right), t \leq s \leq T,
$$

we can find the governing PDE with the Feynman-Kac theorem as

$$
\left\{\begin{array}{c}
\frac{\partial h}{\partial s}+\frac{1}{2}\left(v+\alpha_{s}^{2}\right) \frac{\partial^{2} h}{\partial y^{2}}+\frac{1}{2} \sigma^{2} v \frac{\partial^{2} h}{\partial v^{2}}+\rho \sigma v \frac{\partial^{2} h}{\partial v \partial y} \\
+\left[r-\frac{1}{2}\left(v+\alpha_{s}^{2}\right)\right] \frac{\partial h}{\partial y}+k(\theta-v) \frac{\partial h}{\partial v}=0 \\
\left.h\left(\phi ; s, T, y_{s}, v_{s} \mid X_{T}\right)\right|_{s=T}=e^{j \phi y_{T}} .
\end{array}\right.
$$

Considering the results in Heston (1993), we let $\tau=T-s$ and assume that the solution of $h$ take the form of

$$
h\left(\phi ; s, T, y_{s}, v_{s} \mid X_{T}\right)=e^{C(\phi, \tau)+D(\phi, \tau) v_{s}+j \phi y_{s}},
$$

which is substituted into PDE (2.13) to yield the following two ODEs (ordinary differential equations)

$$
\begin{aligned}
& \frac{\partial D}{\partial \tau}=\frac{1}{2} \sigma^{2} D^{2}+(\rho \sigma \phi j-k) D-\frac{1}{2}\left(j \phi+\phi^{2}\right), \\
& \frac{\partial C}{\partial \tau}=k \theta D+r j \phi-\frac{1}{2}\left(j \phi+\phi^{2}\right) \alpha_{s}^{2} .
\end{aligned}
$$

The ODE for $D(\phi, \tau)$ is a Riccati equation, which can be solved with the techniques presented in Heston (1993). Once we have obtained the analytical solution of $D(\phi, \tau)$, $C(\phi, \tau)$ could be easily figured out by simply integrating on the both sides of the governing 
ODE, the result of which can be specified as

$$
C(\phi, \tau)=j r \phi \tau+\frac{k \theta}{\sigma^{2}}\left\{[d-(j \phi \rho \sigma-k)] \tau-2 \ln \left[\frac{1-g e^{d \tau}}{1-g}\right]\right\}-\int_{s}^{T}\left\langle-\frac{1}{2}\left(j \phi+\phi^{2}\right) \bar{\alpha}^{2}, X_{z}\right\rangle d z .
$$

Therefore, by setting $s=t$, we can obtain the solution of the inner expectation

$$
h\left(\phi ; t, T, v_{t} \mid X_{T}\right)=e^{C(\phi, \tau)+D(\phi, \tau) v_{t}} .
$$

The disappearance of $y_{t}$ results from the fact that $y_{t}=0$.

Clearly, the left work in deriving the conditional forward characteristic function is to work out the outer expectation shown in Equation (2.11), which is now

$$
m\left(\phi ; t, T, v_{0} \mid X_{T}\right)=e^{C(\phi, \tau)} E\left[e^{D(\phi, \tau) v_{t}} \mid v_{0}, X_{T}\right] .
$$

The above equation has demonstrated that the only difficulty in reaching our objective is the expectation with respect the volatility process itself. If we denote

$$
f\left(\phi ; t, T, s, v_{s} \mid X_{T}\right)=E\left[e^{D(\phi, \tau) v_{t}} \mid v_{s}, X_{T}\right],
$$

then for any $s \in[0, t], f\left(\phi ; t, T, s, v_{s} \mid X_{T}\right)$ should satisfy the following PDE

$$
\left\{\begin{array}{l}
\frac{\partial f}{\partial s}+\frac{1}{2} \sigma^{2} v \frac{\partial^{2} f}{\partial v^{2}}+k(\theta-v) \frac{\partial f}{\partial v}=0 \\
\left.f\left(\phi ; t, T, v_{s} \mid X_{T}\right)\right|_{s=t}=e^{D(\phi, \tau) v_{t}}
\end{array}\right.
$$

Similar to the solution procedure illustrated above, we also assume that the solution to PDE (2.20) can be expressed as

$$
f\left(\phi ; t, T, s, v_{s} \mid X_{T}\right)=e^{\bar{C}\left(\phi ; \tau, \tau_{s}\right)+\bar{D}\left(\phi ; \tau, \tau_{s}\right) v_{s}},
$$


which can lead to

$$
\begin{aligned}
& \frac{\partial \bar{D}}{\partial \tau_{s}}=\frac{1}{2} \sigma^{2} \bar{D}^{2}-k \bar{D}, \\
& \frac{\partial \bar{C}}{\partial \tau_{s}}=k \theta \bar{D},
\end{aligned}
$$

with the substitution of the solution into PDE (2.20). Here, $\tau_{s}=t-s$, and the initial conditions are

$$
\bar{D}(\phi ; \tau, 0)=D(\phi, \tau), \quad \bar{C}(\phi ; \tau, 0)=0 .
$$

Both of the two ODEs can be easily solved after some algebraic computation. Therefore, with $s$ being set to zero, we can finally reach our desired result

$$
m\left(\phi ; t, T, v_{0} \mid X_{T}\right)=e^{C(\phi, \tau)} f\left(\phi ; t, T, 0, v_{0} \mid X_{T}\right)=e^{C(\phi, \tau)+\bar{C}(\phi ; \tau, t)+\bar{D}(\phi ; \tau, t) v_{0}} .
$$

This has completed the proof.

With the conditional forward characteristic function of the underlying price, it is not difficult to find that the forward characteristic function is the expectation of $m\left(\phi ; t, T, v_{0} \mid X_{T}\right)$, which can be calculated as

$$
m\left(\phi ; t, T, v_{0}, X_{0}\right)=E\left[e^{j \phi y_{T}} \mid y_{0}, v_{0}, X_{0}\right]=E\left[m\left(\phi ; t, T, v_{0} \mid X_{T}\right) \mid X_{0}\right] .
$$

The expectation in Equation (2.25) is actually with respect to the Markov chain, which can further yield the following result

$$
m\left(\phi ; t, T, v_{0}, X_{0}\right)=e^{\widetilde{C}(\phi ; \tau, t)+\bar{D}(\phi ; \tau, t) v_{0}} E\left[e^{\int_{t}^{T}\left\langle G(s), X_{s}\right\rangle d s} \mid X_{0}\right],
$$

by substituting Equation (2.9) into (2.25). Here, $G(s)=-\frac{1}{2}\left(j \phi+\phi^{2}\right) \bar{\alpha}^{2}$. According to the results in Elliott \& Lian (2013), if $A$ is used to denote the transition rate matrix of the 
Markov chain $X_{t}$, we can obtain

$$
E\left[e^{\int_{t}^{T}\left\langle G(s), X_{s}\right\rangle d s} \mid X_{t}\right]=\left\langle e^{M} X_{t}, I\right\rangle
$$

with $I=(1,1)^{\prime}$, and the matrix $M$ defined as

$$
M=\int_{t}^{T} A^{\prime}+\operatorname{diag}[G(s)] d s
$$

which in our case can be further derived as

$$
M=\left(\begin{array}{cc}
-\frac{1}{2}\left(j \phi+\phi^{2}\right) \alpha_{1}^{2} \tau-\lambda_{12} \tau & \lambda_{21} \tau \\
\lambda_{12} \tau & -\frac{1}{2}\left(j \phi+\phi^{2}\right) \alpha_{2}^{2} \tau-\lambda_{21} \tau
\end{array}\right)
$$

Thus, from the tower rule of the expectation, we have

$$
E\left[e^{\int_{t}^{T}\left\langle G(s), X_{s}\right\rangle d s} \mid X_{0}\right]=E\left\{E\left[e^{\int_{t}^{T}\left\langle G(s), X_{s}\right\rangle d s} \mid X_{t}\right] \mid X_{0}\right\}=E\left[\left\langle e^{M} X_{t}, I\right\rangle \mid X_{0}\right]
$$

On the other hand, if $p_{i j}(t), i=1,2, j=1,2$ are used to denote the transition probability from state $i$ to state $j$ within the time period $[0, t]$, they can be expressed as

$$
\begin{aligned}
& p_{11}(t)=\frac{\lambda_{21}}{\lambda_{12}+\lambda_{21}}+\frac{\lambda_{12}}{\lambda_{12}+\lambda_{21}} e^{-\left(\lambda_{12}+\lambda_{21}\right) t}, \\
& p_{22}(t)=\frac{\lambda_{12}}{\lambda_{12}+\lambda_{21}}+\frac{\lambda_{21}}{\lambda_{12}+\lambda_{21}} e^{-\left(\lambda_{12}+\lambda_{21}\right) t},
\end{aligned}
$$

with $p_{12}(t)=1-p_{11}(t)$ and $p_{21}(t)=1-p_{22}(t)$. With $P$ representing the transition probability matrix

$$
P=\left(\begin{array}{ll}
p_{11}(t) & p_{12}(t) \\
p_{21}(t) & p_{22}(t)
\end{array}\right)
$$


the unknown expectation in Equation (2.26) can be derived as

$$
E\left[e^{\int_{t}^{T}\left\langle G(s), X_{s}\right\rangle d s} \mid X_{0}\right]=\left\langle P b, X_{0}\right\rangle
$$

Here, the vector $b$ is defined as

$$
b=\left(\begin{array}{c}
\left\langle e^{M} X^{1}, I\right\rangle \\
\left\langle e^{M} X^{2}, I\right\rangle
\end{array}\right),
$$

with $X^{1}=(1,0)^{\prime}$ and $X^{2}=(0,1)^{\prime}$. Therefore, the forward characteristic function is finally obtained as

$$
m\left(\phi ; t, T, v_{0}, X_{0}\right)=e^{\widetilde{C}(\phi ; \tau, t)+\bar{D}(\phi ; \tau, t) v_{0}}\left\langle P b, X_{0}\right\rangle
$$

With the forward characteristic function being worked out, the derivation of the prices of variance and volatility swaps becomes quite straightforward, and the results are presented in the following proposition, with $R e[\cdot]$ being used to denote taking the real part of the argument.

Proposition 2.2. If the delivery price of a variance swap, $K_{v a r}$, and that of a volatility swap, $K_{\text {vol }}$, are defined in Equation (2.5), they can be respectively formulated as

$$
K_{v a r}=\frac{100^{2}}{T} \sum_{i=1}^{N}\left[m\left(-2 j ; t_{i-1}, t_{i}, v_{0}, X_{0}\right)-2 m\left(-j ; t_{i-1}, t_{i}, v_{0}, X_{0}\right)+1\right]
$$

and

$$
K_{v o l}=100 \sqrt{\frac{2}{\pi N T}} \int_{0}^{+\infty} \sum_{i=1}^{N} \operatorname{Re}\left[\frac{m\left(\phi-j ; t_{i-1}, t_{i}, v_{0}, X_{0}\right)-m\left(\phi ; t_{i-1}, t_{i}, v_{0}, X_{0}\right)}{j \phi}\right] d \phi .
$$


Proof. The delivery price for a variance swap can be calculated as

$$
\begin{aligned}
K_{v a r} & =\frac{100^{2}}{T} \sum_{i=1}^{N} E\left[\left(\frac{S_{t_{i}}-S_{t_{i-1}}}{S_{t_{i-1}}}\right)^{2}\right]=\frac{100^{2}}{T} \sum_{i=1}^{N} E\left[e^{2 y t_{i}}-2 e^{y_{t_{i}}}+1\right] \\
& =\frac{100^{2}}{T} \sum_{i=1}^{N}\left[m\left(-2 j ; t_{i-1}, t_{i}, v_{0}, X_{0}\right)-2 m\left(-j ; t_{i-1}, t_{i}, v_{0}, X_{0}\right)+1\right],
\end{aligned}
$$

the last step of which is obtained from the definition of the forward characteristic function. The derivation of the volatility swap price is a bit more complicated, the first step of which is to evaluate the expected value of the realized volatility

$$
\begin{aligned}
E\left[\left|\frac{S_{t_{i}}-S_{t_{i-1}}}{S_{t_{i-1}}}\right|\right] & =\int_{0}^{+\infty}\left(e^{y_{t_{i}}}-1\right) p\left(y_{t_{i}}\right) d y_{t_{i}}+\int_{-\infty}^{0}\left(1-e^{y_{t_{i}}}\right) p\left(y_{t_{i}}\right) d y_{t_{i}} \\
& =-\int_{0}^{+\infty} p\left(y_{t_{i}}\right) d y_{t_{i}}+\int_{-\infty}^{0} p\left(y_{t_{i}}\right) d y_{t_{i}} \\
& +\int_{0}^{+\infty} e^{y_{t_{i}}} p\left(y_{t_{i}}\right) d y_{t_{i}}-\int_{-\infty}^{0} e^{y t_{i}} p\left(y_{t_{i}}\right) d y_{t_{i}},
\end{aligned}
$$

where $p\left(y_{t_{i}}\right)$ is the forward density function of $y_{t_{i}}$. According to the relationship between the distribution function and characteristic function, it is not difficult for us to obtain

$$
\int_{0}^{+\infty} p\left(y_{t_{i}}\right) d y_{t_{i}}=\frac{1}{2}+\frac{1}{\pi} \int_{0}^{+\infty} \operatorname{Re}\left[\frac{m\left(\phi ; t_{i-1}, t_{i}, v_{0}, X_{0}\right)}{j \phi}\right] d \phi
$$

It should also be noticed that $\frac{e^{y t_{i}} p\left(y_{t_{i}}\right)}{m\left(-j ; t_{i-1}, t_{i}, v_{0}, X_{0}\right)}$ is a density function of a random variable since

$$
\int_{-\infty}^{+\infty} e^{y_{t_{i}}} p\left(y_{t_{i}}\right) d y_{t_{i}}=m\left(-j ; t_{i-1}, t_{i}, v_{0}, X_{0}\right)
$$

As a result, we can derive the characteristic function of this particular random variable, which is actually the Fourier transform of the density function, i.e.,

$$
\bar{m}\left(\phi ; t_{i-1}, t_{i}, v_{0}, X_{0}\right)=\frac{m\left(\phi-j ; t_{i-1}, t_{i}, v_{0}, X_{0}\right)}{m\left(-j ; t_{i-1}, t_{i}, v_{0}, X_{0}\right)} .
$$


From this, one can again make use of the relationship between the distribution function and characteristic function to calculate the following integral

$$
\int_{0}^{+\infty} \frac{e^{y_{t_{i}}} p\left(y_{t_{i}}\right)}{m\left(-j ; t_{i-1}, t_{i}, v_{0}, X_{0}\right)} d y_{t_{i}}=\frac{1}{2}+\frac{1}{\pi} \int_{0}^{+\infty} \operatorname{Re}\left[\frac{m\left(\phi-j ; t_{i-1}, t_{i}, v_{0}, X_{0}\right)}{j \phi \cdot m\left(-j ; t_{i-1}, t_{i}, v_{0}, X_{0}\right)}\right] d \phi
$$

Therefore, the following expectation can be obtained

$$
E\left[\left|\frac{S_{t_{i}}-S_{t_{i-1}}}{S_{t_{i-1}}}\right|\right]=\frac{2}{\pi} \int_{0}^{+\infty} \operatorname{Re}\left[\frac{m\left(\phi-j ; t_{i-1}, t_{i}, v_{0}, X_{0}\right)-m\left(\phi ; t_{i-1}, t_{i}, v_{0}, X_{0}\right)}{j \phi}\right] d \phi,
$$

and the delivery price of a volatility swap can be expressed as

$$
\begin{aligned}
K_{v o l} & =100 \sqrt{\frac{\pi}{2 N T}} \sum_{i=1}^{N} E\left[\left|\frac{S_{t_{i}}-S_{t_{i-1}}}{S_{t_{i-1}}}\right|\right] \\
& =100 \sqrt{\frac{2}{\pi N T}} \int_{0}^{+\infty} \sum_{i=1}^{N} \operatorname{Re}\left[\frac{m\left(\phi-j ; t_{i-1}, t_{i}, v_{0}, X_{0}\right)-m\left(\phi ; t_{i-1}, t_{i}, v_{0}, X_{0}\right)}{j \phi}\right] d \phi
\end{aligned}
$$

With the completion of the above proof, the closed-form pricing formulae for variance and volatility swaps have been successfully derived under the two-factor stochastic volatility model. The numerical behavior of the two newly derived formulae will be studied in the next section through the designed numerical experiments, with an emphasis being placed on the comparison between the variance and volatility swap prices calculated with our formula and those under the Heston model. This is motivated by the fact that the Heston model is a widely adopted one in practice and the adopted model is a combination of the Heston model and the regime switching Black-Scholes model. Such kind of comparison can demonstrate the influence of introducing the regime switching mechanics. 


\section{$3 \quad$ Numerical experiments and examples}

In this section, numerical experiments are carried out to study the influence of introducing the regime switching factor into the Heston model, which would be conducted through the comparison of the variance and volatility swap prices calculated through our formulae with those obtained under the Heston model with the formula for variance swap prices in Zhu \& Lian (2011) and the formula for volatility swap prices in Zhu \& Lian (2015). In the following, unless otherwise state, the values of the parameters used are listed as follows. The risk-free interest rate $r$ and the volatility of volatility $\sigma$ are set to be 0.05 and 0.1 respectively. The mean reverting speed $k$ and the long-term mean $\theta$ are chosen to be 10 and 0.05 respectively, and the correlation $\rho$ between the underlying price and stochastic volatility is -0.5 . In addition, the initial stochastic volatility level $v_{0}$ is 0.03 , and the sampling frequency $N$, which is measured by the number of times per year, is 4 . The two transition rate, i.e., $\lambda_{12}$ and $\lambda_{21}$, are assumed to be equal to each other, both taking the value of 10 , while the values for the regime switching volatility, $\alpha_{1}$ (state 1) and $\alpha_{2}$ (state 2 ), are set to be 0.01 and 0.1 , respectively.

Before we study the property of the newly derived formulae, their accuracy should be verified in advance to ensure there are no algebraic errors. In order to demonstrate the correctness of our formula, we will compare the variance swap prices calculated through our formula and those obtained with the Monte Carlo simulation. To improve the efficiency of the Monte Carlo simulation, we adopt a semi-Monte-Carlo simulation (Liu et al. 2006) that in one simulation, we first generate a Markov chain so that the $\alpha_{X_{t}}$ becomes a time-dependent parameter, and then calculate the conditional characteristic function (2.9), followed by the calculation of the variance swap price for one simulation by simply substituting the conditional characteristic function into the variance swap price formula (2.36). Finally, we can obtain the Monte Carlo price through repeating the above process and taking the mean of the obtained prices. Variance swap prices corresponding to state 1 calculated through our formula and those from the semi-Monte Carlo simulation are 


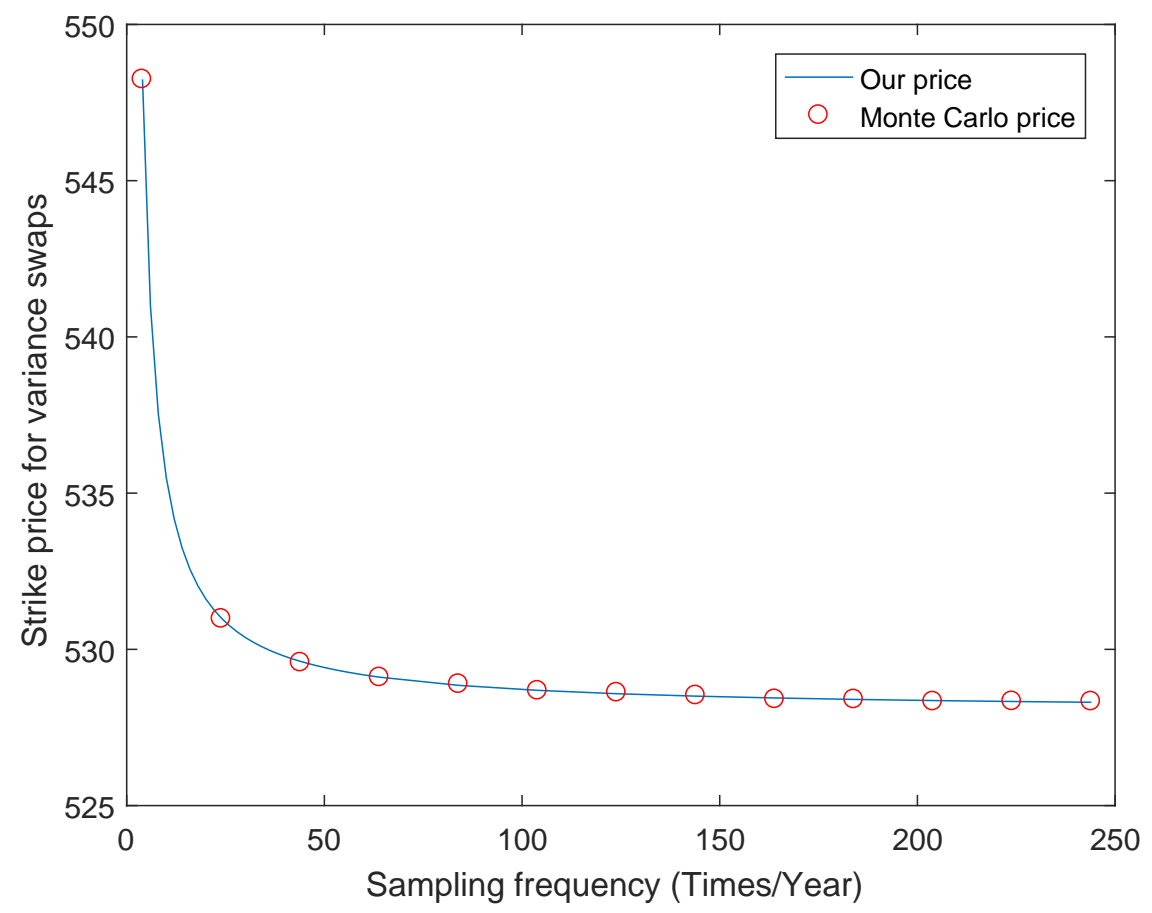

Figure 1: The conparison of variance swap prices from our formula and the Monte Carlo simulation.

presented in Figure 1. It is clear that they are very close to each other with the point-wise relative difference being less than $0.01 \%$, which can certainly demonstrate the accuracy of our formula. Furthermore, prices under both models decrease sharply when the sampling frequency starts to increase from a small value, while the prices remain almost unchanged if we further increase the sampling frequency when it is already very large.

As stated before, the adopted model will degenerate to the Heston model if the constant volatility in each state of the Markov chain equals to zero. Thus, what we will check first is this particular degeneration, which can also partially verify the validity of our formulae. In order to achieve this goal, a scale parameter $z$ varying within the range $[0,1]$ is introduced so that it is a factor of the constant volatility of each state, i.e., $\lambda_{1}=0.01 * z$ and $\lambda_{2}=0.1 * z$. In this case, variance and volatility prices under both models with respect to this scale parameter are presented in Figure 2. As expected, when the values of the volatility controlled by the Markov chain for both states equal to 0 , the two variance 


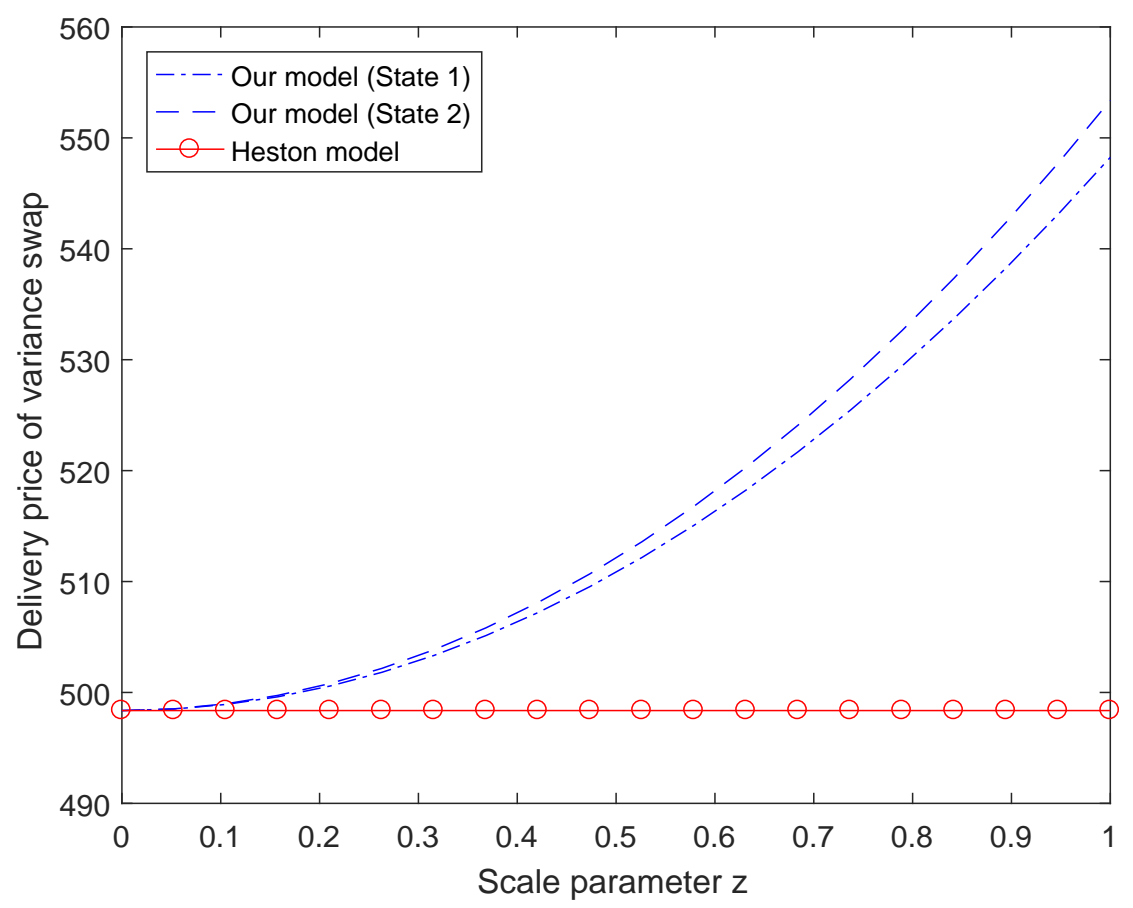

(a) Variance swap prices.

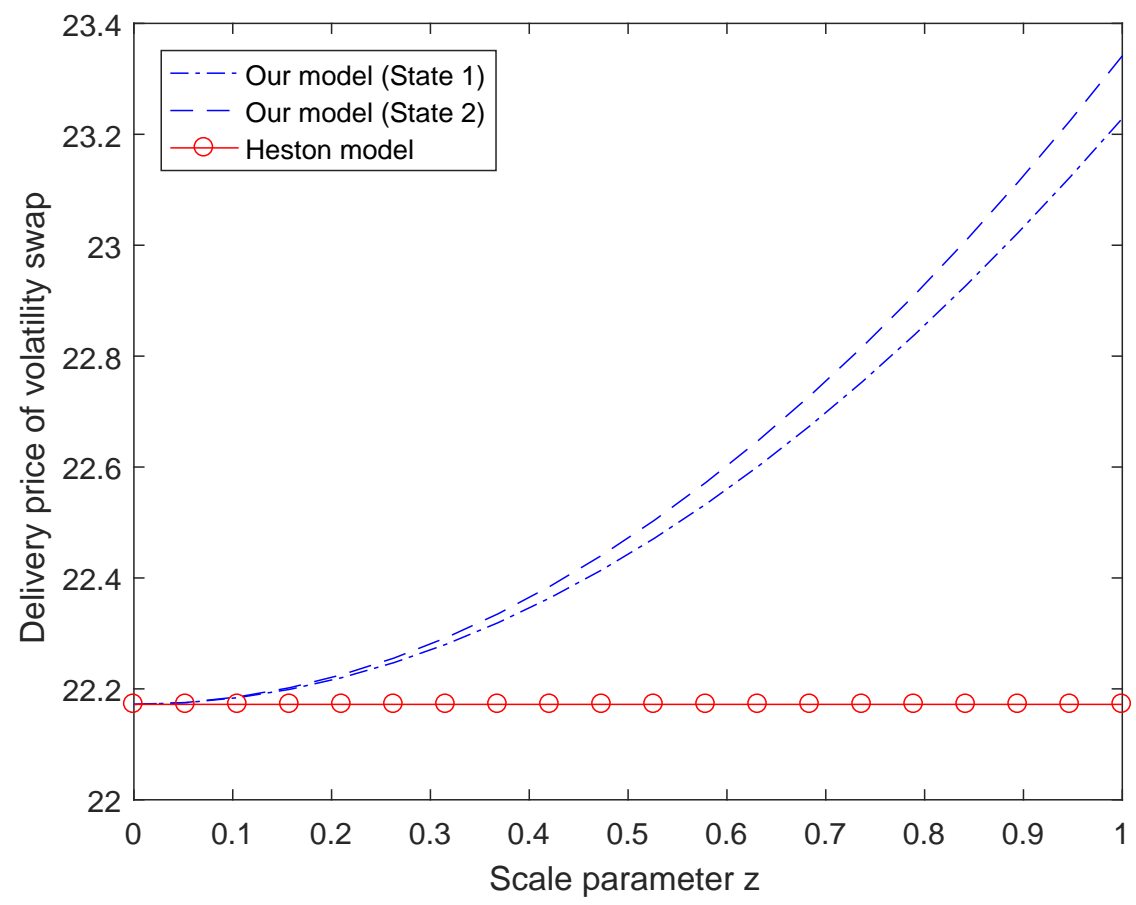

(b) Volatility swap prices.

Figure 2: Delivery prices with or without regime switching with respect to the scale parameter. 
swap prices corresponding to different states no longer differ from each other, and they both equal to the prices under the Heston model, as shown in Figure 2(a). What's more, variance swap prices of both states under our model are monotonic increasing functions of the regime switching volatility, with prices in state 1 being lower than those in state 2 , while they are both larger than prices under the Heston model. This is actually reasonable since the higher the volatility (risk), the larger the derivative price will be. A similar pattern can be observed for volatility swap prices in Figure 2(b), where the volatility swap prices also increase when the level of the regime switching volatility is enlarged. The only difference in the two sub-figures is that the magnitude of the delivery price for the volatility swap is approximately the square root of that for the variance swap, which can be explained by the fact that the realized variance is about the square of the realized volatility.

Depicted in Figure 3 is the comparison of our variance and volatility swap prices with those under the Heston model in terms of different values of the time to expiry. It is clear that prices under both models are increasing functions of the time to expiry when other parameters are kept unchanged, with our prices being always higher than those under the Heston model. Moreover, the increasing speed for state1 and state 2 under our two-factor stochastic volatility model is slightly higher and lower than that in the Heston model, respectively, and the two state prices under our model are quite close to each other when the time to expiry is very large.

Figure 4 displays different delivery prices of variance and volatility swaps against the volatility of state $1, \alpha_{1}$. A similar phenomenon, which has already been shown in Figure 2, can also be observed here that variance and volatility swap prices are monotonic increasing functions of the volatility level for both states. Moreover, prices of state 2 are larger than those of State 1 when the volatility of state 1 is smaller than that of state 2 , while an apposite phenomenon can be observed when the value of the volatility in state 1 is enlarged to be higher than that in state 2 . It should also be remarked that the prices corresponding both states will equal to each other when the volatility of state 1 equals to that of state 2 , 


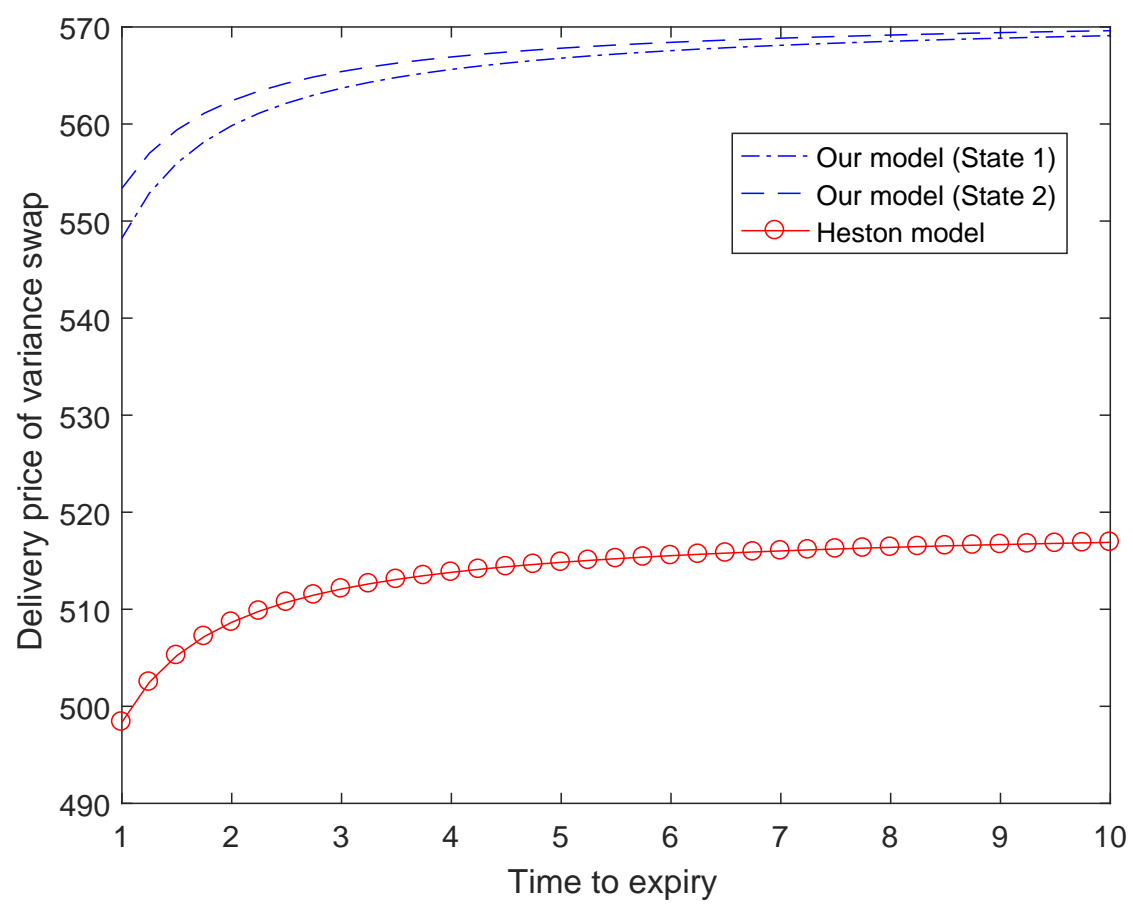

(a) Different variance swap prices with respect to time to expiry.

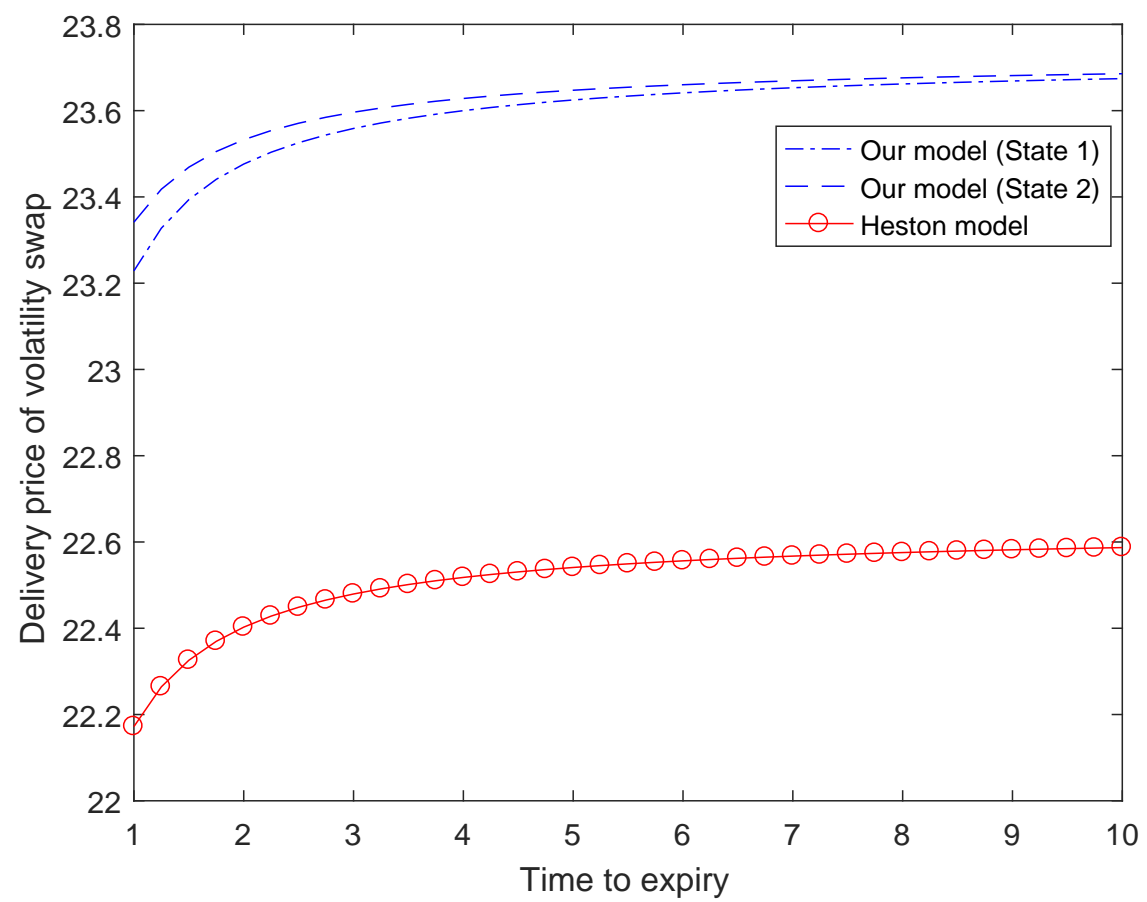

(b) Different volatility swap prices with respect to time to expiry.

Figure 3: Delivery prices with or without regime switching with respect to time to expiry. 


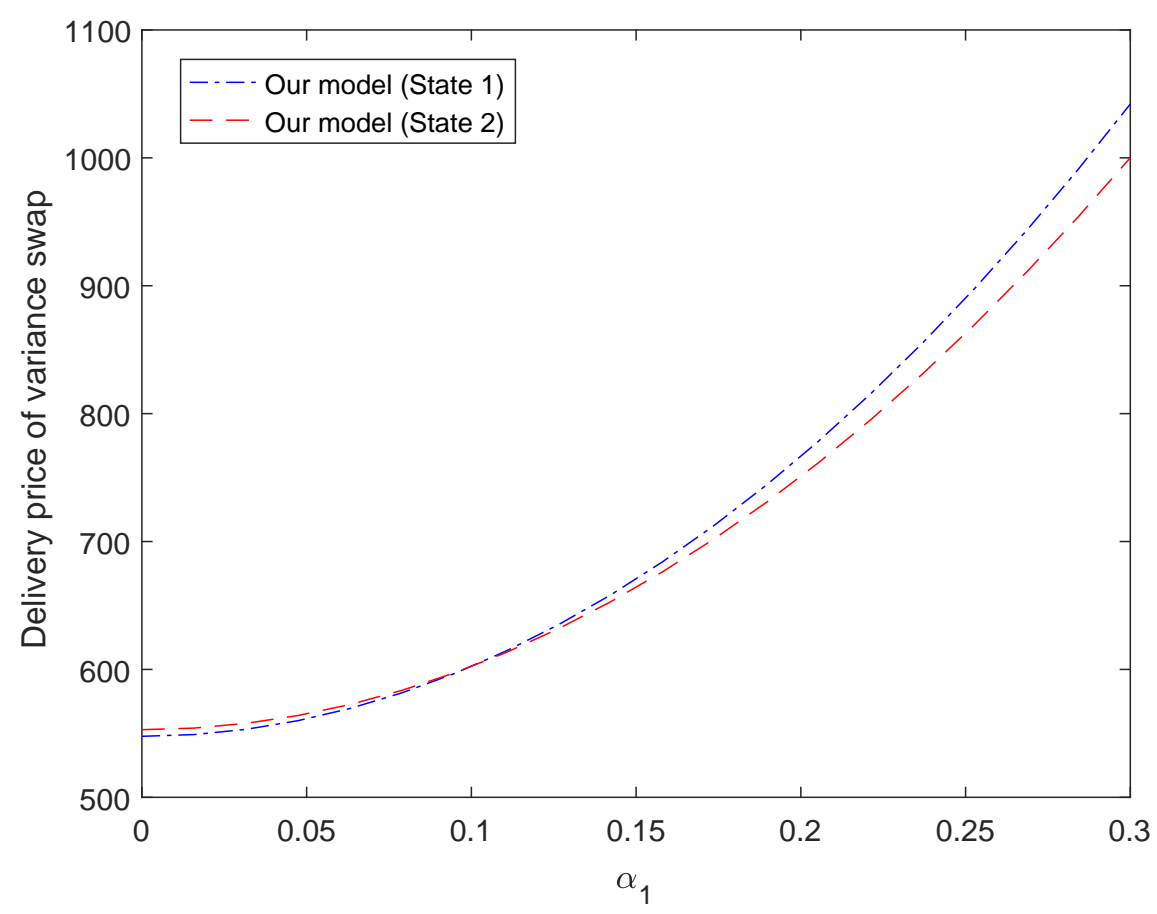

(a) Variance swap prices.

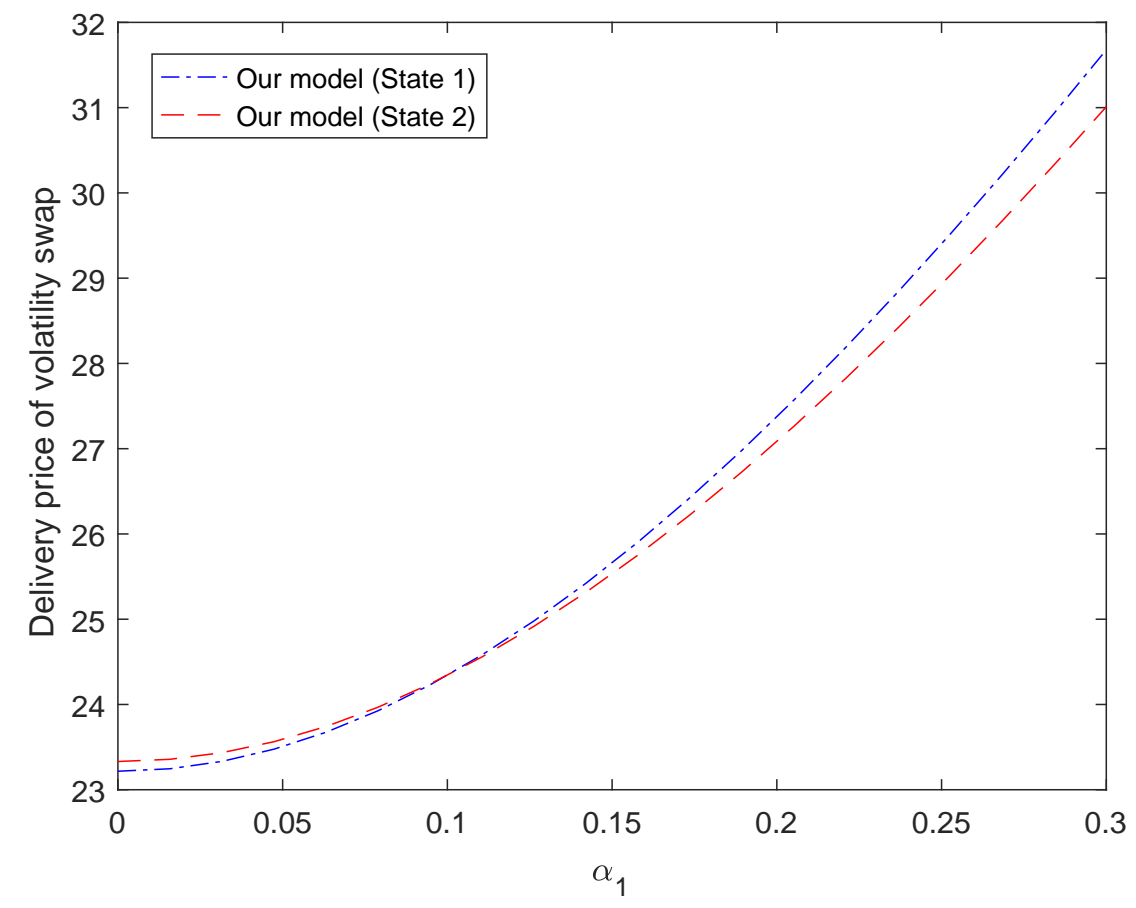

(b) Volatility swap prices.

Figure 4: The effect of $\alpha_{1}$ on the delivery prices of variance and volatility swaps. 
which can be understood by considering the fact that there are no actual regime switching in this case.

What is shown in Figure 5 is the change of delivery prices of variance and volatility swaps with respect to the transition rates. Obviously, prices in state 1 are always lower than those in state 2, no matter the value of the transition rates is. This can be mainly explained that the probability of the Markov chain transiting from state 1 to state 2 within a fixed period equals to that transiting from state 2 to state 1 , as a result of assuming that the transition rates for both states equal to each other, and thus a large volatility level of the initial state tend to yield high prices. Furthermore, both of variance and volatility swap prices are monotonic increasing and decreasing functions of transition rates in state 1 and those in state 2, respectively, which implies that the gap between prices of both states are narrowed down when we increase the transition rates. This is because the probability of the chain transiting from state 1 to state 2 increases with transition rates, and in this case the volatility starting in state 1 (low volatility) has more chance to transfer to state 2 (high volatility), leading to a higher price for state 1 . In contrast, the volatility starting in state 2 (high volatility) has more chance to transfer to state 1 (low volatility), resulting in a lower price when increasing transition rates.

It should be remarked that the calibration of derivative pricing models involving regime switching with real market data is not an easy task, as there are two state prices in our model and we are usually not sure which state the underlying asset belongs to in practice. Fortunately, He \& Zhu (2017) recently proposed a new closed system, in which they assumed that the probability of the underlying price being in each state, instead of the current state, should be known and be regarded as a model parameter. As a result, the model price can be uniquely determined as the weighted average of all state prices. Given that our model also has the same regime switching features, the same approach should be applicable, and thus the unique delivery price of a variance/volatility swap under our model can be determined in a very similar way. 


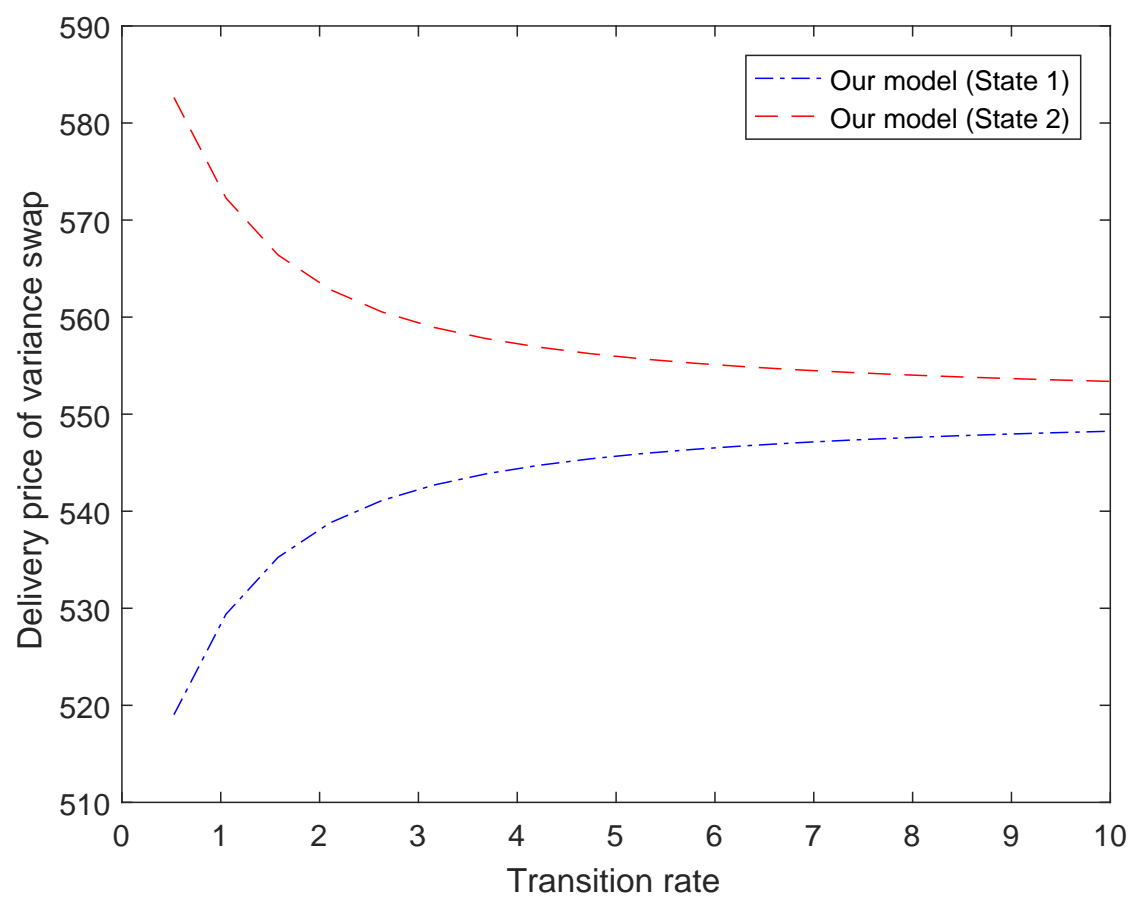

(a) Variance swap prices.

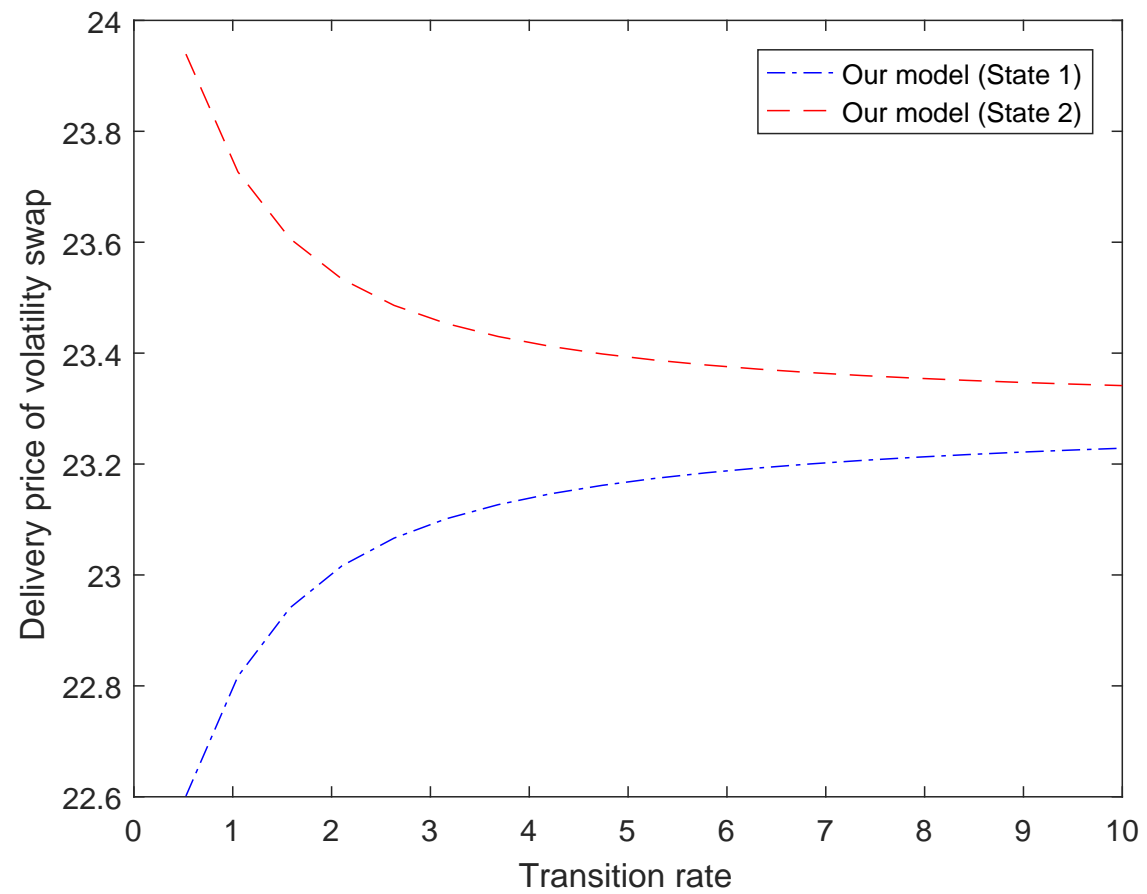

(b) Volatility swap prices.

Figure 5: The effect of the transition rates on the delivery prices of variance and volatility swaps. 


\section{Conclusion}

In this paper, analytical pricing formulae for variance and volatility swaps have been derived when the underlying price follows a two-factor Heston stochastic volatility model, with one factor still being modeled by the CIR model while another being controlled by a Markov

chain. Numerical experiments have also been carried out to compare the delivery prices of the two swaps resulting from the current two-factor model and the original Heston model, and the results have shown that the introduction of the regime switching factor into the Heston model can make a significant difference, as far as variance and volatility swap prices are concerned.

\section{References}

Byelkina, S. \& Levin, A. (2010), Implementation and calibration of the extended affine Heston model for basket options and volatility derivatives, in 'Sixth World Congress of the Bachelier Finance Society, Toronto'.

Carr, P. \& Lee, R. (2007), 'Realized volatility and variance: Options via swaps', Risk 20(5), 76-83.

Carr, P. \& Lee, R. (2008), Robust replication of volatility derivatives, in 'PRMIA award for Best Paper in Derivatives, MFA 2008 Annual Meeting'.

Elliott, R. J., Chan, L. \& Siu, T. K. (2015), 'A dupire equation for a regime-switching model', International Journal of Theoretical and Applied Finance 18(04), 1550023.

Elliott, R. J. \& Lian, G.-H. (2013), 'Pricing variance and volatility swaps in a stochastic volatility model with regime switching: discrete observations case', Quantitative Finance 13(5), 687-698. 
Eraker, B. (2004), 'Do stock prices and volatility jump? Reconciling evidence from spot and option prices', The Journal of Finance 59(3), 1367-1403.

Grünbichler, A. \& Longstaff, F. A. (1996), 'Valuing futures and options on volatility', Journal of Banking 6 Finance 20(6), 985-1001.

Habtemicael, S. \& Sengupta, I. (2016a), 'Pricing covariance swaps for BarndorffNielsen and Shephard process driven financial markets', Annals of Financial Economics $\mathbf{1 1}(03), 1650012$.

Habtemicael, S. \& SenGupta, I. (2016b), 'Pricing variance and volatility swaps for Barndorff-Nielsen and Shephard process driven financial markets', International Journal of Financial Engineering 3(04), 1650027.

Hamilton, J. D. (1990), 'Analysis of time series subject to changes in regime', Journal of Econometrics 45(1), 39-70.

He, X.-J. \& Zhu, S.-P. (2016), 'An analytical approximation formula for European option pricing under a new stochastic volatility model with regime-switching', Journal of Economic Dynamics and Control 71, 77-85.

He, X.-J. \& Zhu, S.-P. (2017), 'How should a local regime-switching model be calibrated?', Journal of Economic Dynamics and Control 78, 149-163.

He, X.-J. \& Zhu, S.-P. (2018), 'On full calibration of hybrid local volatility and regimeswitching models', Journal of Futures Markets 38(5), 586-606.

Heston, S. L. (1993), 'A closed-form solution for options with stochastic volatility with applications to bond and currency options', Review of Financial Studies 6(2), 327-343.

Howison, S., Rafailidis, A. \& Rasmussen, H. (2004), 'On the pricing and hedging of volatility derivatives', Applied Mathematical Finance 11(4), 317-346. 
Issaka, A. \& SenGupta, I. (2017), 'Analysis of variance based instruments for OrnsteinUhlenbeck type models: swap and price index', Annals of Finance 13(4), 401-434.

Javaheri, A., Wilmott, P. \& Haug, E. G. (2004), 'GARCH and volatility swaps', Quantitative Finance 4(5), 589-595.

Kun, F. (2014), 'Pricing options under two-factor Markov-modulated stochastic volatility models', Chinese Journal of Applied Prob-ability and Statistics 30(6), 620-630.

Liu, R., Zhang, Q. \& Yin, G. (2006), 'Option pricing in a regime-switching model using the fast Fourier transform', International Journal of Stochastic Analysis 2006.

Salvi, G. \& Swishchuk, A. V. (2014), 'Covariance and correlation swaps for financial markets with markov-modulated volatilities', International Journal of Theoretical and Applied Finance 17(01), 1450006.

Siu, T. K., Yang, H. \& Lau, J. W. (2008), 'Pricing currency options under two-factor Markov-modulated stochastic volatility models', Insurance: Mathematics and Economics 43(3), 295-302.

Swishchuk, A. (2004), 'Modeling of variance and volatility swaps for financial markets with stochastic volatilities', WILMOTT Magazine 2, 64-72.

Swishchuk, A. (2006), 'Modelling and pricing of variance swaps for multi-factors stochastic volatilities with delay', Canadian Applied Mathematics Quarterly 14(4), 439-467.

Zhu, S.-P. \& Lian, G.-H. (2011), 'A closed-form exact solution for pricing variance swaps with stochastic volatility', Mathematical Finance 21(2), 233-256.

Zhu, S.-P. \& Lian, G.-H. (2012), 'An analytical formula for VIX futures and its applications', Journal of Futures Markets 32(2), 166-190.

Zhu, S.-P. \& Lian, G.-H. (2015), 'Analytically pricing volatility swaps under stochastic volatility', Journal of Computational and Applied Mathematics 288, 332-340. 